\title{
Local Existence and Uniqueness Theorem for a Nonlinear Schrödinger Equation with Robin Inhomogeneous Boundary Condition
}

\author{
Charles Bu \\ Department of Mathematics, Wellesley College, Wellesley, MA, USA \\ Email:cbu@wellesley.edu
}

How to cite this paper: $\mathrm{Bu}, \mathrm{C}$. (2020) Local Existence and Uniqueness Theorem for a Nonlinear Schrödinger Equation with Robin Inhomogeneous Boundary Condition. Journal of Applied Mathematics and Physics, 8, 464-469.

https://doi.org/10.4236/jamp.2020.83036

Received: February 12, 2020

Accepted: March 7, 2020

Published: March 10, 2020

Copyright (c) 2020 by author(s) and Scientific Research Publishing Inc. This work is licensed under the Creative Commons Attribution International License (CC BY 4.0).

http://creativecommons.org/licenses/by/4.0/

\section{Open Access}

\begin{abstract}
In recent years, a vast amount of work has been done on initial value problems for important nonlinear evolution equations like the nonlinear Schrödinger equation (NLS) and the Korteweg-de Vries equation (KdV). No comparable attention has been given to mixed initial-boundary value problems for these equations, i.e. forced nonlinear systems. But in many cases of physical interest, the mathematical model leads precisely to the forced problems. For example, the launching of solitary waves in a shallow water channel, the excitation of ion-acoustic solitons in a double plasma machine, etc. In this article, we present the PDE (Partial Differential Equation) method to study the following $i u_{t}=u_{x x}-g|u|^{p} u, g \in R, p>3, x \in \Omega=[0, L], 0 \leq t<\infty \quad$ with initial condition $u(x, 0)=u_{0}(x) \in H^{2}(\Omega)$ and Robin inhomogeneous boundary condition $u_{x}(0, t)+\alpha u(0, t)=R_{1}(t), t \geq 0$ and $u_{x}(L, t)+\alpha u(L, t)=R_{2}(t), t \geq 0$ (here $\alpha$ is a real number). The equation is posed in a semi-infinite strip on a finite domain $\Omega$. Such problems are called forced problems and have many applications in other fields like physics and chemistry. The main tool of PDE method is semi-group theory. We are able to prove local existence and uniqueness theorem for the nonlinear Schrödinger equation under initial condition and Robin inhomogeneous boundary condition.
\end{abstract}

\section{Keywords}

Nonlinear, Schrodinger, Equation, Inhomogeneous, Robin, Boundary,

Condition, Existence and Uniqueness, Classical Solution

\section{Introduction}

This paper is the continuation of an earlier one [1] where local existence and uniqueness theorem was presented for a one-dimensional nonlinear Schrödinger 
equation with initial condition and Dirichlet type inhomogeneous boundary condition on a finite domain $\Omega=[0, L]$. As we know, many physically important nonlinear evolution equations in one spatial and one temporal dimensions have been found to possess exact solution by the method of inverse scattering transform (IST). For example, the IST technique has been applied to Cauchy problems in the infinite interval $-\infty<x<\infty$ for decaying [2], periodic [3] and self-similar potentials [4]. When a boundary condition is imposed, sometimes it is called forced problem [5] [6] [7] [8]. The physical importance of forced problems for the nonlinear integrable systems was discussed by Kaup [9]. Existence and uniqueness of the solution to the Korteweg-de Vries equation for $0 \leq x, t<\infty$ where $u(x, 0)$ and $u(0, t)$ are given have been proven by Bona and Winther [10] [11].

The following nonlinear Schrödinger equation (NLS) posed in the quarter plane with Dirichlet inhomogeneous condition ( $k$ is a real constant) has been studied by the author:

$$
\begin{gathered}
i u_{t}=u_{x x}+k|u|^{2} u \\
u(x, 0)=u_{0}(x), u(0, t)=Q(t) .
\end{gathered}
$$

The initial condition $u_{0}(x),-\infty<x<\infty$ and inhomogeneous boundary condition $Q(t), t \geq 0$ are imposed. Existence and uniqueness of a global classical solution were proved via PDE method provided that the initial-boundary data are "nice" (cf. [12]). Further, (1.1) is shown to be well-posed [13].

For the NLS posed in the quarter plane with Robin inhomogeneous condition $(k \in \mathrm{R})$ :

$$
\begin{gathered}
i u_{t}=u_{x x}+k|u|^{2} u \\
u(x, 0)=u_{0}(x), u_{x}(0, t)+\alpha u(0, t)=R(t)
\end{gathered}
$$

similar results were available [14] [15].

Solving such problems has important physical and mathematical implications. For example, (1.2) arises in the propagation of the optical solitons [16]. Also, the NLS with an additional term $u_{x}$ on the right-hand side and $\alpha \rightarrow \infty$ models water waves [17].

This paper will investigate a more general version of nonlinear Schrödinger equation $i u_{t}=u_{x x}-g|u|^{p} u$ on a semi-infinite strip $x \in \Omega=[0, L], 0 \leq t<\infty$. Robin type inhomogeneous boundary conditions are imposed on both endpoints. Using PDE method, we will prove the existence of a unique local classical solution.

\section{Existence and Uniqueness of the Local Solution}

In this paper, we study the following NLS with initial condition and Robin inhomogeneous boundary condition $(g \in \mathrm{R}, p>3))$ :

$$
\begin{gathered}
i u_{t}=u_{x x}-g|u|^{p} u, x \in \Omega=[0, L], 0 \leq t<\infty \\
u(x, 0)=u_{0}(x) \in H^{2}(\Omega) \\
u_{x}(0, t)+\alpha u(0, t)=R_{1}(t)
\end{gathered}
$$




$$
u_{x}(L, t)+\alpha u(L, t)=R_{2}(t)
$$

here $\alpha$ is a real number and $t>0$. Using semigroup technique we prove that there exists a unique classical local solution.

We shall utilize the following notations and assume that $\alpha$ is an arbitrary real number throughout.

$$
\begin{gathered}
Q_{1}(t)=u(0, t), P_{1}(t)=u_{x}(0, t) \\
R_{1}(t)=P_{1}(t)+\alpha Q_{1}(t) \\
Q_{2}(t)=u(L, t), P_{2}(t)=u_{x}(L, t) \\
R_{2}(t)=P_{2}(t)+\alpha Q_{2}(t) \\
R_{0}=\sum_{i=1}^{i=2} \sup _{0 \leq t \leq T}\left(\left|R_{i}(t)\right|+\left|R_{i}^{\prime}(t)\right|\right)
\end{gathered}
$$

We assume that $u_{0}(x) \in H^{2}(\Omega), R_{1}(t) \in C^{1}(\Omega), R_{2}(t) \in C^{1}(\Omega)$ have appropriate smoothness. In addition, they satisfy the necessary compatibility conditions to ensure the existence of solution at $\partial \Omega$ and $t=0$, i.e.

$u_{x}(0,0)+\alpha u_{0}(0)=R_{1}(0)$ and $u_{x}(L, 0)+\alpha u_{0}(L)=R_{2}(0)$.

Lemma 2.1. Let $A=-i D_{x}^{2}+i a$, $D(A)=\left\{v: v \in L^{2}, v_{x x} \in L^{2}, v^{\prime}(0)+\alpha v(0)=v^{\prime}(L)+\alpha v(L)=0\right\}$. Then the operator $A$ is the infinitesimal generator of a continuous semigroup of contractors $N(t)=\exp A t$ for $t \geq 0$. Here $a$ is an appropriate positive constant depending on $\alpha$.

Proof. Let $X=\left\{v: v \in L^{2}(\Omega), v_{x x} \in L^{2}(\Omega)\right\}$. Then $X$ is a Banach space with a norm equivalent to $H^{2}(\Omega)$-norm. Let $H=L^{2}(\Omega)$,

$V=\left\{v \in H^{1}(\Omega): v^{\prime}(0)+\alpha v(0)=0=v^{\prime}(L)+\alpha v(L)\right\}$ then $D(A)$ and $V$ are dense in $H$. From Gagliardo-Nirenberg estimates (cf. [18] for details), we have $\|u\|_{0, \infty} \leq c\|u\|_{2,2},\left\|u^{\prime}\right\|_{0, \infty} \leq c^{\prime}\|u\|_{2,2}$. Let $v_{n} \in D(A), A v_{n} \rightarrow y, v_{n} \rightarrow z$ in $H$. Then clearly $\left\{v_{n}\right\}$ is a Cauchy sequence in $X$. Completeness of $X$ implies that $\left\{v_{n}\right\}$ converges in $X$ and $A z=y$. Also,

$$
\begin{aligned}
\left|z^{\prime}(0)+\alpha z(0)\right| & \leq\left|v^{\prime}(0)+\alpha v(0)\right|+\left|z^{\prime}(0)-v^{\prime}(0)\right|+|\alpha||z(0)-v(0)| \\
& \leq c^{\prime}\|z-v\|_{X}+c|\alpha|\|z-v\|_{X} \rightarrow 0
\end{aligned}
$$

Similarly

$$
\left|z^{\prime}(0)+\alpha z(0)\right| \leq c^{\prime}\|z-v\|_{X}+c|\alpha|\|z-v\|_{X} \rightarrow 0
$$

This shows that $z \in D(A)$. Thus $A$ is closed. To show that the resolvent set of $A$ contains $R^{+}$, let $v \in V$. Consider

$$
\begin{aligned}
((\lambda-A) v, v) & =\int_{0}^{L}\left(\lambda v-i a v+i v_{x x}\right) \bar{v} \mathrm{~d} x \\
& =(\lambda-i a) \int_{0}^{L}|v|^{2} \mathrm{~d} x+i \int_{0}^{L} v_{x x} \bar{v} \mathrm{~d} x \\
& =(\lambda-i a)\|v\|_{2}^{2}+\left.i v^{\prime} \bar{v}\right|_{0} ^{L}-i\left\|v^{\prime}\right\|_{2}^{2} \\
& =(\lambda-i a)\|v\|_{2}^{2}-i\left\|v^{\prime}\right\|_{2}^{2}+i v^{\prime}(L) \bar{v}(L)-i v^{\prime}(0) \bar{v}(0) \\
& =(\lambda-i a)\|v\|_{2}^{2}-i\left\|v^{\prime}\right\|_{2}^{2}+i \alpha|v(0)|^{2}-i \alpha|v(L)|^{2}
\end{aligned}
$$


Take the imaginary part of (2.2),

$$
|((\lambda-A) v, v)| \geq\left.\left|a\|v\|_{2}^{2}-\alpha\right| v(0)\right|^{2}+\alpha|v(L)|^{2}+\left\|v^{\prime}\right\|_{2}^{2} \mid
$$

By [18], there exists $c>0$ such that

$$
|v(0)|+|v(L)| \leq 2\|v\|_{\infty} \leq 2 c\left\|v^{\prime}\right\|_{2}^{\frac{1}{2}}\|v\|_{2}^{\frac{1}{2}} .
$$

Then (2.3) becomes

$$
\begin{aligned}
|((\lambda-A) v, v)| & \geq a\|v\|_{2}^{2}-2|\alpha| c^{2}\left\|v^{\prime}\right\|_{2}\|v\|_{2}+\left\|v^{\prime}\right\|_{2}^{2} \\
& \geq a\|v\|_{2}^{2}-2 \alpha^{2} c^{4}\|v\|_{2}^{2}-\frac{1}{2}\left\|v^{\prime}\right\|_{2}^{2}+\left\|v^{\prime}\right\|_{2}^{2} \\
& =\left(a-2 \alpha^{2} c^{4}\right)\|v\|_{2}^{2}+\frac{1}{2}\left\|v^{\prime}\right\|_{2}^{2}
\end{aligned}
$$

If one sets $a>2 \alpha^{2} c^{4}$ then

$$
|((\lambda-A) v, v)| \geq c_{0}\left(\|v\|_{2}^{2}+\left\|v^{\prime}\right\|_{2}^{2}\right)=c_{0}\|v\|_{V}^{2}
$$

By Theorem 2.3.3 of [19], for $\lambda>0$ the operator $\lambda-A$ maps $D(A)$ 1-1 onto $H$. Now let $v \in D(A)$. For $\lambda>0$, by taking the real part of (2.2), one has the following inequality $\lambda\|v\|_{2} \leq\|(\lambda-A) v\|_{2}$ thus $\left\|(\lambda-A)^{-1}\right\| \leq \frac{1}{\lambda}$. By Hille-Yosida Theorem (cf. [20]), the unbounded and linear operator $A$ is the infinitesimal generator of a continuous semigroup of a contractions $N(t)=\exp$ At for $t \geq 0$.

Theorem 2.2 Local Existence-Uniqueness. For $R_{i}(t) \in C^{2}(\Omega), i=1,2$, $u_{0}(x) \in H^{2}(\Omega)$, there exists a unique classical solution $u$ for Equation (2.1) such that $u \in C^{1}\left(\left[0, T_{M}\right), D(A)\right) \cap L^{2}\left(\left[0, T_{M}\right), D(A)\right)$ with either $\lim \|u\|_{D(A)}=\infty$ as $t \rightarrow T_{M}$ or $T_{M}=\infty$.

To prove this theorem, we first apply the following transformation:

$$
\begin{aligned}
u(x, t) & =v(x, t)+S(x, t) \\
& =v(x, t)+\frac{(L-x)^{2}}{L(\alpha L-2)} R_{1}(t)+\frac{x^{2}}{L(\alpha L+2)} R_{2}(t)
\end{aligned}
$$

Substituting (2.4) into (2.1) yields

$$
v_{t}=-i v_{x x}+i a v+G(v, x, t)
$$

where

$$
\begin{gathered}
G=-i a v+i g|v+S|^{p}(v+S)-S_{t}-i S_{x x} \\
v_{x}(0, t)+\alpha v(0, t)=v_{x}(L, t)+\alpha v(L, t)=0, v \in H^{2}(\Omega)
\end{gathered}
$$

One can converts (2.5) to an integral equation:

$$
v=N(t) v_{0}+\int_{0}^{t} N(t-s) G(s) \mathrm{d} s
$$

By similar analysis as in [12], $G$ is locally Lipschitz in $V$ under the norm of $D(A)$ uniformly on $[0, T]$ and for each $v \in D(A), G$ is continuous from $[0, T]$ into $D(A)$ (note $S \in C^{1}[0, T]$ for any $T$ ). Thus one can use Theorem 
6.1.7 in [20] to obtain the local existence-uniqueness theorem for (2.5) with $v \in C^{1}\left[0, T_{M}\right)$ with either $\lim \|u\|_{D(A)}=\infty$ as $t \rightarrow T_{M}$ or $T_{M}=\infty$. Since $u=v+S$ and $S \in H^{2}(\Omega)$ uniformly on $[0, T]$ for any $T>0$, we conclude immediately that $u$ satisfies (2.1) and our existence and uniqueness are proved.

\section{Conclusion}

Famous nonlinear partial differential equations like nonlinear Schrödinger equation have important applications when the boundary value is not zero. For such equation posed in a semi-infinite strip, we used PDE method to prove that there exists a unique classical local solution, via semigroup theory. The PDE method presented in this paper to study the NLS is an approach different from the IST method in [7]. Along with [12], we try to provide some answers to the questions raised in [9]. There are some further research that could be done in this area. For example, we believe that well-posedness of (2.1) is a subject of research. Also, the existence of a global solution is an open problem. We will continue our work and report any further advances.

\section{Acknowledgements}

This research was supported by the William R. Kenan Jr. Professorship, a Brachman Hoffman Small Grant and a Wellesley College Faculty Award.

\section{Conflicts of Interest}

The author declares no conflicts of interest regarding the publication of this paper.

\section{References}

[1] Bu, C. (2020) Local Existence and Uniqueness Theorem for a Nonlinear Schrodinger Equation with Dirichlet Inhomogeneous Boundary Data, to Appear.

[2] Ablowitz, M.J. and Segur, H. (1981) Solitions and the Inverse Scattering Transform. Studies in Applied Mathematics, North Holland. https://doi.org/10.1137/1.9781611970883

[3] Zakharov, V.E. and Manakov, S.V. (1980) Theory of Solitions. The Inverse Method, Nauka, Moscow.

[4] Its, A.R. and Novokshenov, V.Y. (1985) The Isomonodromic Deformation Method and the Theory of Painleve Equations. In: Lecture Notes in Mathematics 1191, Springer, Berlin. https://doi.org/10.1007/BFb0076661

[5] Carroll, R. (1990) On the Forced Nonlinear Schrodinger Equation. Japan Journal of Industrial and Applied Mathematics, 7, 321-344. https://doi.org/10.1007/BF03167847

[6] Carroll, R. and Oharu, S. (1990) Some Remarks on the Forced Kortweg-de Vries Equation. Applicable Analysis, 39, 83-93. https://doi.org/10.1080/00036819008839973

[7] Fokas, A.S. (1989) Initial-Boundary Value Problem for the Nonlinear Schrodinger Equation. Physica D, 35, 167-185. https://doi.org/10.1016/0167-2789(89)90101-2 
[8] Fokas, A. and Ablowitz, M. (1989) Forced Nonlinear Evolution Equations and the Inverse Scattering Transform. Studies in Applied Mathematics, 80, 253-272. https://doi.org/10.1002/sapm1989803253

[9] Kaup, D.J., Rogers, C. and Moddie, T.B. (1984) Wave Phenomena. North-Holland, Amsterdam.

[10] Bona, J. and Winther, R. (1983) The Korteweg-de Vries Equation, Posed in a Quarter-Plane. SIAM Journal on Mathematical Analysis, 6, 1056-1106. https://doi.org/10.1137/0514085

[11] Bona, J. and Winther, R. (1989) The Korteweg-de Vries Equation in a Quarter-Plane, Continuous Dependence Result. Differential and Integral Equations, 2, 228-250.

[12] Carroll, R. and Bu, C. (1991) Solutions of the Forced Nonlinear Schrodinger Equation (NLS) Using PDE Techniques. Applicable Analysis, 41, 33-51. https://doi.org/10.1080/00036819108840015

[13] Bu, C. (1992) On Well-Posedness of the Forced Nonlinear Schrodinger Equation. Applicable Analysis, 46, 219-239. https://doi.org/10.1080/00036819208840122

[14] Bu, C. (1994) An Initial-Boundary Value Problem for the Nonlinear Schrodinger Equation. Applicable Analysis, 53, 241-255. https://doi.org/10.1080/00036819408840260

[15] Bu, C. (2000) Forced Cubic Schrödinger Equation with Robin Boundary Data: Continuous Dependency Result. Journal of the Australian Mathematical Society, Series $B, 41,301-311$. https://doi.org/10.1017/S0334270000011255

[16] Kodama, Y. (1985) Optical Soliton in a Monomode Fifiber: Transportation and Propagation in Nonlinear Systems. Journal of Statistical Physics, 39, 597-614. https://doi.org/10.1007/BF01008354

[17] Newell, A.C. (1985) Solitons in Mathematics and Physics. SIAM, Philadelphia, PA. https://doi.org/10.1137/1.9781611970227

[18] Nirenberg, L. (1959) On Elliptic Partial Differential Equations. The Annali della Scuola Normale Superiore di Pisa, 13, 115-162.

[19] Carroll, R. (1969) Abstract Methods in PDE. Haper Row, New York.

[20] Pazy, A. (1983) Semigroups of Linear Operators and Applications to PDE. Springer, New York. https://doi.org/10.1007/978-1-4612-5561-1 